\title{
Modeling Life
}

\author{
Michael L. Shuler \\ Biomedical Engineering, Cornell University, Ithaca, NY 14853, USA \\ (Received 16 February 2012; accepted 3 April 2012; published online 17 April 2012)
}

Associate Editor K. A. Athanasiou oversaw the review of this article.

\begin{abstract}
We seek to construct physical and mathematical models of life. Such models allow us to test our understanding of how living systems function and how they respond to human imposed stimuli. One system is a genomically and chemically complete model of a minimal cell. This cell is a hypothetical bacterium with the fewest number of genes possible. Such a minimal cell provides a platform to ask about the essential features of a living cell and forms a platform to investigate "synthetic biology." A second system is "Body-on-a-Chip" which is a microfabricated microfluidic system with cells or tissue constructs representing various organs in the body. It can be constructed from human or animal cells and used in drug discovery development. That model is a physical representation of a physiologically based pharmacokinetic model. Both the computer and the physical models provide insight into the underlying biology and provide new tools to make use of that understanding to provide benefits to society.
\end{abstract}

\section{INTRODUCTION}

Models provide a framework in which to think about a system, specifically the interactions within the system and its response to perturbations from outside the system. Building models is a common exercise from bioengineers. Here, I describe two projects which originate in our interest in building models of life itself. The first project focuses on a model of a minimal bacterial cell to test the completeness of our understanding of what is essential for a cell to be alive. This understanding could lead to advances in synthetic biology and human-designed cells. The second is the combined mathematical models of the body, cell culture, and micro fabrication to construct a surrogate for animal or human clinical trials to test the efficiency and

Address correspondence to Michael L. Shuler, Biomedical Engineering, Cornell University, Ithaca, NY 14853, USA. Electronic mail: mls50@cornell.edu

Based on 2011 Pritzker Lecture. safety of drug(s). While these two projects differ greatly in scale, they both employ a common thought process.

\section{MODEL OF A MINIMAL BACTERIAL CELL}

Building a mathematical model of a living cell has been a passion of mine since I was a graduate student at Minnesota in the early 1970s. My ideas were built on the basis of those first articulated by Aris, Fredrickson, and Tsuchiya, ${ }^{26}$ who first formulated the concepts of structured/unstructured and segregated/non-segregated models. However, this work was focused on populations of microbial cells. Structured models introduced the concept that quantitative changes in the constituents of a cell would lead to changes in metabolic activities of the population. What I thought was missing and yet important was to model individual cells and then assemble a model of a population as an ensemble of individual cell models. Such an approach facilitates a detailed model of cellular function (beyond metabolism and avoids an error that existed for the first 10 years of structured population models ${ }^{8}$ ).

The first attempt was the Cornell single cell model of Escherichia coli. This cell model was the first single cell model that did not impose artificial constraints on the system. The external medium composition was set at an initial value. The initial composition of the cell and its size and shape specified. Then the differential equations in the model were integrated forward in time. The resulting cell composition, size, shape and growth rate were an output of the model and responded explicitly to changes in the external nutrient levels. An important feature of this modeling strategy was the inclusion of biological mechanisms to control chromosome replication, cell division, and changes in cell geometry. Thus, the model is much more than a metabolic model but contains "events" such as initiation of DNA replication and cell division. An event is a 
discrete change in cell state. The initial model formulation $^{6,16}$ was expanded to include a much larger array of components including plasmids encoding synthesis of recombinant proteins ${ }^{1,10}$ and interaction of plasmid encoded functions and cellular metabolism. ${ }^{11}$ The E. coli model has always had the capability of linking genomic structure with cellular response. With the advent of systems biology, large scale linkage becomes possible. We have added genomic detail for nucleic acids $^{4}$ and lipid synthesis ${ }^{3}$ in the context of a $E$. coli model. In these cases, we asked what would be the required minimum number of genes to allow sustained growth in a medium with appropriate performed nutrients. Thus using the coarse grain E. coli model as a host platform we demonstrated that a pathway with 12 genes for nucleotide metabolism would yield sustained replication. ${ }^{4}$ For lipid synthesis, five genes and appropriate preformed lipid precursors would sustain replication identifying. ${ }^{3}$

This modular approach to construction of a minimized genome has the advantage that it allows testing of potential genome constructs in a context that almost assures a stable model cell. The disadvantage is that it is a laborious approach for constructing a candidate minimal cell.

Thus, the basic E. coli model was coarse grain, but it allowed for prediction of cell size, composition, shape, cell cycle parameters, and response to a wide variety of perturbations in external nutrient concentrations and temperature. By incorporating genomic information, it is possible to directly relate physiology to changes in genetic construction. ${ }^{4,11}$

However, we wanted to design a microbe based on a totally defined chromosome containing the minimum number of genes. While this has been a goal of synthetic biology, we were interested in understanding not only the minimum chromosome but also the essential minimum regulatory network that would allow a sustained growth. Such a model could guide the process of constructing such a minimal cell. In this article, we draw a distinction between a minimal cell (i.e., the fewest number of genes) and a minimized cell where all the genetic information not essential to a predetermined function (e.g., production of valuable protein from a plasmid) is deleted. We have discussed the later approach of "biotechnology platform cell" for efficient production of a biological product. ${ }^{7}$ However, for this article, we focus on a minimal cell.

Our strategy to construct a minimal cell is to use the formalisms we used to construct the Cornell coarse grain E. coli model, but to "delump" all model components to explicit chemical compounds. It should be recognized that this remains a whole cell model with "events" and not simply a metabolic model. While several minimal gene sets have been proposed, we adopted one proposed by Gil et al. ${ }^{9}$ We believe this approach has yielded a workable candidate model for construction of a minimal cell.

Atlas and Shuler ${ }^{2}$ have constructed a model cell with 241 genes, 408 chemical species, 36 "events," and 1,176 reaction parameters. We have tested the resulting model for its ability to sustain growth in a benign medium that is chemically defined. The reaction parameter values are numerous, but heavily constrained. At steady-state the size of the cell is double of that at birth (on average) and the chemical composition is unchanged. Thus, if the initial composition of the cell is known, if the initial size is known, and the doubling time is estimated, then the reaction parameters in most reactions can be estimated. Also all concentrations of all chemicals must be greater than zero at all times. These are powerful constraints and allow us to "guess" initial conditions that will give rise to sustain growth and oscillation. The initial "guess" must be good enough to get into a feasible range. Through an interactive process we are able to find a set of feasible parameters. Changing the growth rate changes absolute values of parameters, but the relative ratios of parameters appears to control whether a sustainable steady state can be found. Since the composition of most bacteria and their size and growth rate are known, making initial guesses is fairly easy. Because some of the reactions (e.g., consumption of nucleotides for DNA) are not continuous and are nonlinear, some of the initial guesses must be modified. Nonetheless, finding a candidate set of workable parameters is easier than one might naively guess for such a large number of parameters.

The simulations demonstrate that we can construct a model where replication of the candidate minimal cell can be sustained indefinitely. For a minimal cell, all genes must be essential, so the knockout of a gene should render the model incapable of sustained replication. For essentially all genes in the candidate cell model, the gene knockout renders the cell nonfunctional. A few genes (e.g., DNA repair) could not be tested directly as the model had no mechanism for DNA damage, but we still considered such a gene as essential since physical/chemical forces will lead to DNA damage if such an experimental model were constructed. These calculations, and others, all demonstrate how the model can probe the design features of a cell.

The model did show modest robustness in that a $25 \%$ reduction in enzyme levels (and hence maximum enzymatic reaction rate) was tolerated and a new steady state could be obtained. However, a $60 \%$ reduction was non-recoverable. This result demonstrates that the model structure and intrinsic regulatory structure could be constructed with the expectation that the resulting cell would be stable. 
To construct a minimal cell, we chose multifunction transporters, so as to reduce gene number. For example, the Nat transporter imports arginine, histidine, and lysine. If the external medium is flooded with 15 times the basal amount of arginine in the external medium, then through competitive inhibition, insufficient lysine enters the cell leading to cell death. This observation is an example of how the external environment and the structure of the cell interact. Whether a minimal cell can be sustained is context dependent.

Overall we have shown for the first time that it is possible to build a genomically and chemically complete model of a simple (minimal) cell capable of indefinite growth and replication. Reconstructing life in vitro, based on guidance from such a computer model, is plausible although an experimental demonstration has not been accomplished and such a goal raises ethical issues.

Building a detailed genomically and chemically complete model of a very simple cell is possible. It is not easy to believe it could be done for more complex cells as there are simply too many unknown genes and functions. Such a detailed model for humans is inconceivable; yet a model for humans would be of significant practical benefit. Can we model humans at a useful level?

\section{LIVING CELL MODELS OF HUMANS}

We have combined computer models of the human body (physiologically based-pharmacokineticpharmacodynamic or PBPK-PD model) with microfabricated models based on the PBPK and using living cells in each "compartment." Each compartment corresponds to a tissue or organ in the PBPK and the body. We call this approach a micro cell culture analog or "Body-on-a-Chip"17,22 and was the first description of a multi-compartment cell-containing system that attempted to arrange the various "organ" compartments in a physiologically realistic manner.

We believe that this approach may become a useful addition to the drug development paradigm. Animal studies in preclinical trials are expensive, often take a long time, may raise ethical issues and most importantly do not predict human response very well due to the differences in physiology and biochemistry between species. Currently only 1 in 10 drugs that enter human trials exit as approved drugs. This statistic confirms the poor prediction made by animal models as all 10 passed animal trials and suggests the nature of the opportunity. If successes in clinical trails improved to 1 in 4 , it would cut the cost of drug development significantly and provide more useful drugs to society. Thus a human based mimic, such as "Body-on-a-Chip," that could improve predictions of which drugs are both safe and effective would be valuable. It need not be perfect as even an increase in positive outcomes from clinical trials to 1 in 4 will yield important economic and societal benefits.

We believe that the "Body-on-a-Chip" technology could be the breakthrough technology that could lead to a realistic human surrogate. Indeed recent calls from proposals from DARPA, NIH, and DTRA reflect a national effort (ca. 140 million dollars in aggregate from NIH and DARPA) to build on the Body-on-aChip concept to construct increasingly more sophisticated micro physiological systems for rapid preclinical drug screening.

The basic concept for a "Body-on-a-Chip" device is shown in Fig. 1. The concept is based on a physical analog to physiologically based pharmacokineticpharmocodynmaic (or PBPK-PD) model of the human body to predict the time-dependent concentration of a parental compound (typically a drug) and its metabolites in each compartment. For example, the differential equations that are used in the model to describe reactions in a particular organ compartment are replaced by living cells (either a single cell type or in a more sophisticated model with a tissue engineered construct with multiple cell types in appropriate numbers and spatial arrangement). The organ/tissue compartments are interconnected in the same way as the compartments are linked in the PBPK-PD model. Fluid flow to each compartment and the size of the compartment are in the same ratio as in the human body which should result in similar rates of reaction. We called the first device of this type a cell culture analog (CCA) and was described in Sweeny et al. ${ }^{24} \mathrm{We}$ then constructed our first micro scale device, a microCCA and described it in 2004. ${ }^{17}$ The micro scale makes it feasible to run multiple units relatively cheap.

The design criteria to emulate a PBPK include:

(1) Physiologically realistic ratio of cell mass from one tissue to another

(2) Mimic the flow split of blood during recirculation of a blood surrogate

(3) Residence time (volume/flow rate) in an "organ/tissue" compartment is realistic

(4) The fluid flow generates shear rates within a physiologic range in each tissue compartment.

(5) The ratio of free liquid to cells in each compartment should be physiologic, and

(6) The biologic response of cells in a compartment is authentic; here sophisticated tissue constructs may be critical.

The devices built by our group do a reasonable job satisfying these first four criteria. Our initial devices used cell lines and 2D culture, and the ratio of free liquid to cells was higher than physiologic. For more 


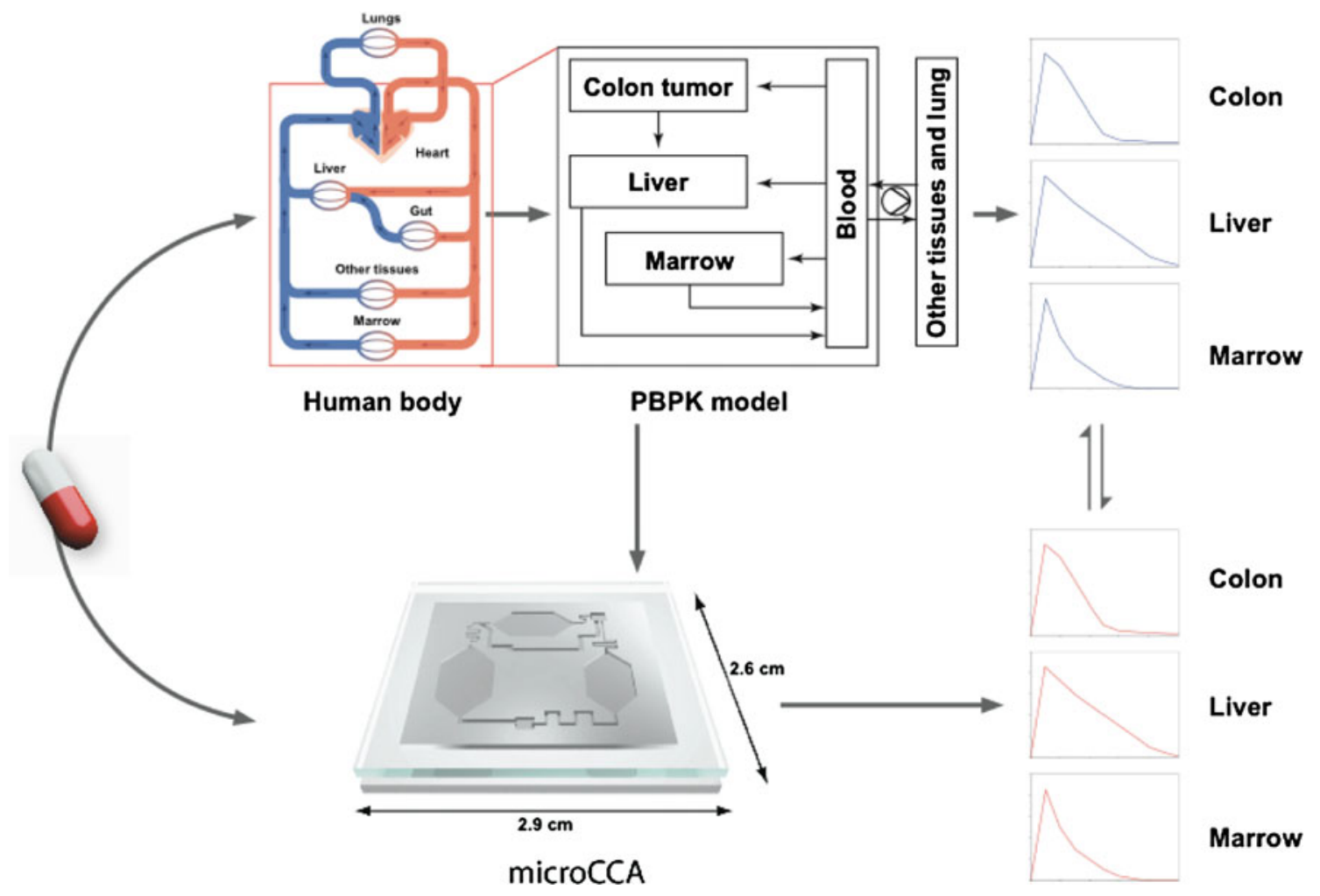

FIGURE 1. The basic concept for a microCCA or "Body-on-a-Chip" is depicted. Drugs administered to the human body and metabolites are distributed throughout the body and their time-dependent distribution can be predicted using a PBPK model. Using the micro-CCA culture equations can be used to replace by living cells or tissue constructs. These devices also provide a basis to measure the time-dependent concentration of the drug and its metabolites. A comparison of the predictions from the PBPK with the experimental results from a microCCA can be used to test the potential veracity of the equations and assumptions used.

recent devices ${ }^{20}, 3 \mathrm{D}$ tissue constructs based on hydrogel entrapped cells have been used, and this technique facilitates design of compartments with more physiologic ratios of liquid to cells.

The last issue of authentic physiological response of cells or tissues in a compartment is the most problematic. While we have used $3 \mathrm{D}$ cultures, these are from cell lines, which do not contain the same mix of cell types as in a real tissue, and for the cell types, they need to be in spatial contact similar to that in the tissue. Our most realistic tissue construct is the GI tract which contains cell lines that mimic three cell types in appropriate ratios. ${ }^{12-14}$ A major component of the current call for proposals from DARPA, NIH, and DTRA is for more biologically authentic tissues. Colleagues with in-depth expertise in tissue engineering have much to contribute in this regard.

A microCCA or "Body-on-a-Chip" device offers several advantages over current technology even when the cell system in a compartment is not fully authentic. Unlike other in vitro devices for evaluating human response to drugs and chemicals, our device mimics realistic dose dynamics as would be appropriate in a PBPK-PD model. Further, a direct comparison of experimental results from a microCCA to predictions by the PBPK model should give the same results if the underlying mechanisms are understood. With the experimental system, we know flow rates, residence time, number of cells, levels of key enzymes in cells, and drug concentrations. Hence, it should be possible to predict and compare results. Thus, using PBPK models and experimental results, the investigator should be able to obtain a deeper insight into possible underlying mechanisms. ${ }^{19,20}$

Another advantage of the microCCA approach is the extrapolation of response of animals to humans. For example, rat tissue can be used in a microCCA and tested with a drug; the predicted results can then be confirmed with animal studies. If the two studies agreed it would provide confidence that the cell culture analog with this drug could make reasonable predictions. If the same type of experiment were done with the microCCA using human tissue, then the prediction for human response with the same drug would have increased credibility if the same approach had worked with another species. Further, differences uncovered between responses at the microCCA level would point to intrinsic species-specific differences.

There are, of course, challenges in making a microCCA a realistic model. This device was the first micro device to be operated for an extended period 
(96 h) with full retention of biological activity and mechanical operation. Because of the very small volume of fluid, evaporation is a potential problem. Even modest evaporation could be problematic due to an increase in the osmolarity of the remaining solution and its effect on cells. Further out-gassing creases bubbles, and bubbles recirculated through the system cause massive cell death. A key solution is to use a vented reservoir in the recirculation loop. The vent is capped with a membrane, such as as polymethylpentene. which allows for good gas exchange, but is relatively impermeable to water vapor. Also a bubble trap ${ }^{21}$ can be built into the system. Operating time has been restricted to $96 \mathrm{~h}$ due to the buildup of metabolic waste products (urea, NH3, and lactate), although this limitation can be removed through the use of partial periodic medium exchange (e.g., 25\% per day) or the use of dialysis.

Another limitation is that a common blood surrogate must be used, which is compatible with all cell types and has the appropriate transport properties for test drugs and chemicals. Serum-containing medium can mimic many of the properties of blood in terms of its carrying capacity, but serum makes mask some of the responses that one may want to observe. The other issue with serum is that it is chemically undefined and may be inconsistent in composition. A serum-free medium with serum albumin and a defined lipid mix maintains may be of the characteristics of blood in terms of carrying capacity. Also there are serum-free defined media that will support long-term culture of difficult to culture cell types. ${ }^{15}$

Another constraint, but not a strong one with the development of a broad range of analytic devices, is analysis based on relatively few cells. The current microCCA has $>10,000$ cells per compartment which provides sufficient material for diagnosis, but requires the sacrifice of the unit. On line analysis either optically ${ }^{18}$ or electrically ${ }^{15}$ allows readout without having to sacrifice the unit and is compatible with measurement of time course.

The major constraint is the authenticity of the biological construct. Ideally these constructs mimic the biological response of tissues. Since all tissues have multiple cell types in predetermined spatial arrangement this constraint is fairly severe. Maintenance of not only chemical, but also mechanical and electrical function is often important. If the fabrication of the device is on typical silicon wafers the depth of the construct should be under $200 \mu \mathrm{m}$; because diffusion in a tissue is typically limited to that distance, this physical constraint is not a strong limitation.

The design and assembly of one such system is shown in Fig. 2. The chip is typically about $2.5 \mathrm{~cm}$ by $2.5 \mathrm{~cm}$ in size and the flow channels are order of $20-100 \mu \mathrm{m}$ wide. Flow is laminar and well behaved.
The design of the device is based on the Hagen PoiseuilleLaw which relates fluid flow and pressure drop with the geometry of the conduit. Using this approach, the fluid velocity in each channel and the liquid residence time in each chamber (which controls the extent of reaction) can be calculated. As shown in the insert in Fig. 2, the calculated and measured values are close. Details of design and operation are given elsewhere. ${ }^{17,22,25,28}$

The system has been used to determine response to naphthalene as a model toxicant. Naphthalene is not toxic itself, but activated in the liver to a reactive form, which causes cell death in other organs, such as the lung. By combining the CCA and a PBPK, we have shown that napthaquinone is the reactive metabolite that causes lung cell death rather than naphthalene epoxide.

We have applied the microCCA concept to evaluating combination therapy for cancer treatment. ${ }^{25}$ Multidrug resistant (MDR) cancer is problematic for treatment. It typically arrives when the cancer is subjected to a chemotherapeutic agent (which often causes significant shrinkage in the tumor). A small subpopulation of cancer cells develop resistance to the drug; while multiple mechanisms of resistance exist the most common is probably the over expression of the membrane pump protein, P-glycoprotein. In MDR cells, this protein is often expressed at $50-100$ fold higher concentrations than in normal, sensitive cancer cells. Consequently the pump intercepts many chemotherapeutic drugs and pumps them back out of the cell before they reach the cytosol. Thus, the cancer cells cannot respond to a therapeutic level of the drug.

A potential solution is to use drugs which inhibit the activity of P-glycoprotein. While such drugs exist, none has exited clinical trials as an approved drug due to unacceptable side effects. It has been postulated that a combination of MDR suppressors with different side effects might yield a useful combination of drugs to allow for effective entry of the drug into the tumor's cells with acceptable side effects. We constructed a microCCA with liver, bone marrow, and uterine cancer cell lines. ${ }^{25}$ The liver represents metabolism of the drugs and generates metabolites that may be physiologically important. The marrow represents the doselimiting response to the MDR suppressing compounds and chemotherapeutic agents. The MDR suppressors may make the body, especially the marrow, more sensitive than normal to the chemotherapeutic drug. Normal cells contain some P-glycoprotein as a protection against harmful compounds ingested by the body, and suppressors may make normal tissue more sensitive to the chemotherapeutic drug. For the uterine cancer module, two chambers (side-by-side) are used. One contains a parental cell line derived from uterine cancer, while the second represents a derivative cell line 
(a)

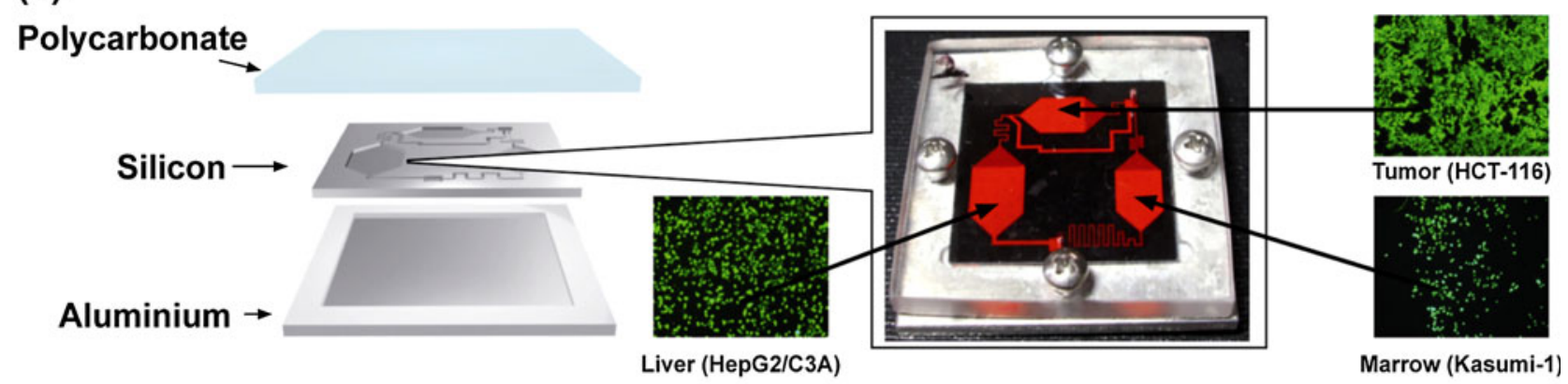

(b)
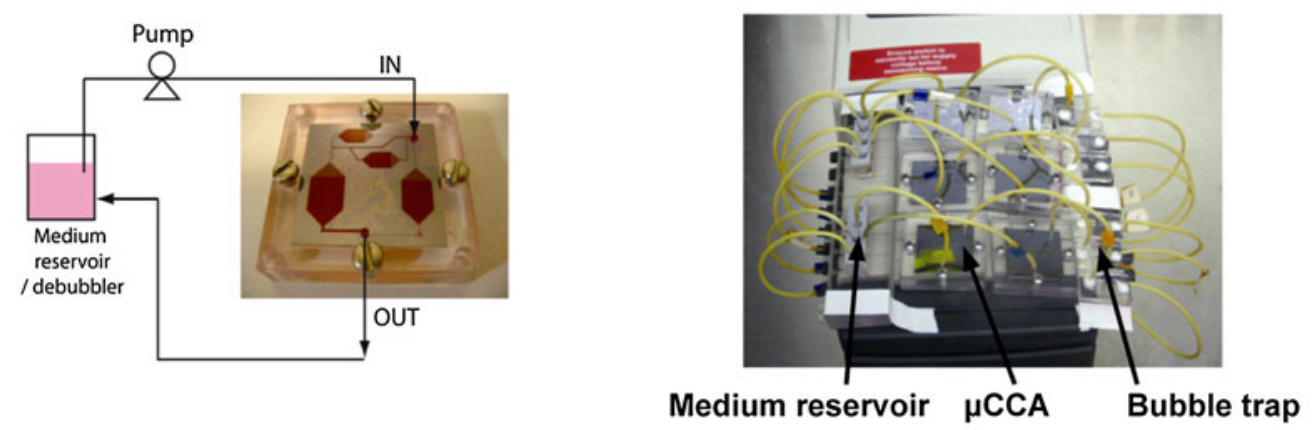

(c)

\begin{tabular}{|l|c|c|c|}
\hline & Tumor & Liver & Marrow \\
\hline Calculation & & 52 & 128 \\
Residence time (s) & 70 & 161 & 54 \\
Velocity (um/s) & 72 & & \\
Measurement & & $50 \pm 4$ & $137 \pm 3$ \\
Residence time (s) & $70 \pm 4$ & $168 \pm 6$ & $59 \pm 1$ \\
Velocity (um/s) & $69 \pm 2$ & \\
\hline
\end{tabular}

FIGURE 2. Depiction of a microCCA and aspects of its operation. (a) Shows the construction of such a device and the seeding of cells in a hydrogel onto the device. (b) Shows the chip connected to an external reservoir that mimics the non-reactive, nonabsorptive tissues of the body. (c) Compares predicted values of flow to every compartment and the residence time of an element of fluid in the chamber to its measure of value.

that presumably differs only in the over expression of P-glycoprotein.

For the experiments, doxorubicin was chosen as the chemotherapeutic drug. Two P-glycoprotein suppressors were chosen: cyclosporine (immune suppressor) and nicardipine (a $\beta$-channel blocker). When applied to the microCCA the MDR suppressing capabilities of the cyclosporine and nicardipine were confirmed. The response of the system to a near therapeutic level of doxorubicin demonstrated selective killing effect on cancer cells versus liver and marrow. Killing was dosedependent. Each MDR-suppressing compound led to reduced viability of the P-glycoprotein over expressing uterine cancer cell line. However, the surprising result was that the killing of the MDR-resistant cell line was increased through a synergistic interaction of the two MDR suppressing drugs. Such synergism was observed only in the device with its dynamic exchange of metabolites and not in comparison tests done in multiwell plates. Thus, this device and approach not only mimic aspects of human response to drugs (e.g., doxorubicin) but also predict responses that may lead to new therapeutic strategies. No human clinical data exist to test the predicted synergistic effect, but the in vitro device does suggest that such an approach may be useful.

We also have used the microCCA concept to test another combination therapy for cancer. Here, we have made the technological advance of using 3D constructs rather than monolayer systems. This allows the fluid to cell ratio in a chamber become more physiologic and potentially allow cells to be used at closer to tissue-like densities. While these studies only used cell lines embodied in hydrogels, the technique can be applied with any tissue engineered construct. The authenticity of the tissue analog in the compartment will ultimately be critical to effective predictions. 
This system was described by Sung and Shuler ${ }^{20}$ who addressed the issue of a combination treatment for colon cancer. It contain modules for "liver" (to mimic metabolism), "colon cancer" (target), marrow (sensitive cell line), and "other" (no cells, but representing flow through non-reactive, non-absorbing tissues). The drug was Tegafur, a prodrug for 5-flurouracil (5 FU). The $\mathrm{p} 450 \mathrm{~s}$ in the liver are required to convert Tegafur into 5-FU. The 5-FU damages DNA, RNA, and thymidylate synthase by displacing uracil and preventing further polymerization. However, $5 \mathrm{FU}$ has a relatively short life span in the circulation due to catabolism by the enzyme, dihydropyrimidine dehydrogenase (DPD). Uracil is a competitive inhibitor for the action of DPD on 5-FU; addition of $4 \mathrm{~mol}$ of uracil to one of a Tegafur is optimal in rodent experiments for tumor reduction.

We compared the response of our system to multiwell plate assays using colon cancer cells as a target. In the microCCA, Tegafur and 5-FU were nearly equally effective in killing colon cells and the addition of uracil increased cell killing. These observations are consistent with clinical observations. However, in the multiwell plate format, only 5-FU killed cells and Tegafur was ineffective (as expected due to the lack of significant p450 activity in the colon cancer cells). This study confirmed that a microCCA could reveal realistic prediction of clinical responses that would not be obvious from standard multiwell plate assays.

More recently, ${ }^{19}$ we have demonstrated that the external pump in the above system can be removed, and a "pumpless" chip used. Using the same cell lines and compounds, we demonstrated that a similar system using gravity as a motive force would give results that compare well to the system with an external pump. A rocker platform was used to provide defined changes in relative fluid heights between two reservoirs, and given known cross-sectional areas of the conduits, the effective flow rate and residence times could be predicted.

The above examples relied on models of only the systemic circulation. However, barrier tissues, such as the gut, lung, skin, etc. modulate access of external chemicals to the systemic circulation. To model the effects of oral uptake of drugs and chemicals, we have made models of the GI tract connected to the systemic circulation.

We have constructed models of the GI tract that contain multiple cell types and functionality. The current model contains up to three cell types: an early passage Caco-2 intestinal epithelial cell line, HT29MTX which will form a goblet-like cell and produces a mucus, and Raji B lymphocytes that when cocultured with Caco-2cells forms a M-cell (or manifold cell) that is involved in the uptake of particles. A mucus layer influences uptake of nutrients and chemicals. ${ }^{14}$ The GI tract model has been coupled with systemic circulation to observe interaction of the GI tract with a liver compartment in terms of acetaminophen toxicity. ${ }^{13}$ The presence of the GI tract modulated the amount of acetaminophen delivered to the liver. Predictions of response to high concentration of acetaminophen were similar to that observed in rodent studies. These studies demonstrate our ability to create micro-fabricated GI tract models that contain multiple cell types and provide reasonable predictions of in vivo responses.

The above model is based on the use of a flat membrane. The human GI tract has macrovilli as well as microvilli. We have constructed micro-fabricated macrovilli using collagen and then coated the surface with intestinal cells demonstrating a 3D GI tract model. ${ }^{23}$ We believe that we can fabricate the macrovilli with "blood vessels" inside them by adapting a technique developed by Stroock et al..$^{5}$ Also, we have developed a micro-fabrication technique using SU-8 and silicon pillars to form macrovilli. With controlled pore size ranging from 0.5 to $3.0 \mu \mathrm{m}$ and high porosity, we have demonstrated that we can grow Caco-2 cells on the membrane (submitted). This technique currently supports macrovilli of a $100-\mu \mathrm{m}$ height, while the collagen-based technique will support macrovilli of $250-500 \mu \mathrm{m}$ in height which is more physiological for much of the small intestine. Thus, we have developed the basic fabrication techniques to model macrovilli and have demonstrated co-culture of relevant cell types.

We have used our basic GI tract module to examine response to the oral uptake of dietary iron in the presence and absence of nanoparticles that may also be ingested. ${ }^{12}$ Our in vitro experiments show that $50 \mu \mathrm{m}$ polystyrene, carboxylated, spherical particles are transported via a paracellular, non-energy-dependent process while $200 \mathrm{~nm}$ particles of the same material transport are transported across the in vitro GI tract through an energy-dependent process such as within cellular vesicles. When nanoparticles are present at moderate concentrations iron transport is inhibited, potentially making an individual go from an ironsufficient to an iron-deficient diet. The human in vitro prediction was compared to studies we did using chicks. In that study, we showed that the in vitro predictions were consistent with the acute (short term) response of the chicks. However, the chronic (or longterm response) differs because the chicks increase the size of the macrovilli and consequently surface area to compensate for the presence of nanoparticles (Mahler et al. $^{12}$ ). These experiments suggest the potential utility of the in vitro predictions.

Although much remains to be done to build a fully predictive in vitro/PBPK human model that is robustly accurate, our studies have made significant advances 
toward that goal. Coupling that technology with improved biological models of tissues and organs will lead to human surrogates that will speed drug discovery and reduce-dependence on animal models. It brings us closer to models that will help us understand the biological bases of human life.

\section{CONCLUSION}

In this article, I have briefly reviewed two different types of biological models. Each is quantitative within a mathematical framework. Each is deeply informed by experimental results. Indeed, in the "Body-on-aChip" project, the model is primarily experimental but guided by a mathematical model. Both projects demonstrate how an engineering approach may offer new insights into understanding and manipulating biological systems.

\section{ACKNOWLEDGMENTS}

I owe much to my former students who worked on these projects. I also wish to thank the selection committee for being given the opportunity to present the Pritzker Lecture; it was a great honor to be selected.

\section{REFERENCES}

${ }^{1}$ Ataai, M. M., and M. L. Shuler. A mathematical model for prediction of plasmid copy number and genetic stability in Escherichia coli. Biotechnol. Bioeng. 30:389-397, 1987.

${ }^{2}$ Atlas, J., and M. L. Shuler. Modeling a minimal cell. In: Methods of Molecular Biology Microbial Systems Biology, Microbial Systems Biology: Methods and Protocols, Chap. 21, edited by A. Navid. New York, NY: Humana Press, 2012.

${ }^{3}$ Castellanos, M., K. Keiichiro, S. K. Lai, and M. L. Shuler. A genomically/chemically complete module for synthesis of lipid membrane in a minimal cell. Biotechnol. Bioeng. 97(2):397, 2007.

${ }^{4}$ Castellanos, M., D. B. Wilson, and M. L. Shuler. A modular minimal cell model: purine and pyrimidine transport and metabolism. Proc. Natl Acad. Sci. USA 101(17):6681-6686, 2004.

${ }^{5}$ Cross, V. L., Y. Zheng, N. W. Choi, S. S. Verbridge, B. A. Sutermaster, L. J. Bonassar, C. Fischbach, and A. D. Stroock. Dense type 1 collagen matrices that support cellular remodeling and microfabrication for studies of tumor angiogenesis and vasculogenesis in vitro. Biomaterials 31:8596-8607, 2010.

${ }^{6}$ Domach, M. M., S. K. Leung, R. E. Cahn, G. G. Cocks, and M. L. Shuler. Computer model for glucose-limited growth of a single cell of Escherichia coli B/r-A. Biotechnol. Bioeng. 26:203-216, 1984.
${ }^{7}$ Foley, P., and M. L. Shuler. Considerations for the design and construction of a synthetic platform cell for biotechnological applications. Biotechnol. Bioeng. 105:26-36, 2010.

${ }^{8}$ Fredrickson, A. G. Formulation of structured growth models. Biotechnol. Bioeng. 18:1481-1485, 1976.

${ }^{9}$ Gil, R., F. J. Silva, J. Peveto, and A. Moya. Determination of the core of a minimal bacterial gene set. Microbiol. Mol. Biol. Rev. 68:518-537, 2004.

${ }^{10}$ Kim, B.-G., and M. L. Shuler. Analysis of pBR322 replication kinetics and its dependency on growth rate. Biotechnol. Bioeng. 36(3):233-242, 1990.

${ }^{11}$ Laffend, L. A., and M. L. Shuler. Ribosomal protein limitations in Escherichia coli under conditions of high translational activity. Biotechnol. Bioeng. 43:388-398, 1994.

${ }^{12}$ Mahler, G. J., M. B. Esch, E. Tako, T. L. Southand, S. D. Archer, R. P. Glahn, and M. L. Shuler. Oral exposure to polystyrene nanoparticles effects iron absorption. Nat. Nanotechnol. (published online), 2012. doi:10.1038/ nnano.2012.3.

${ }^{13}$ Mahler, G. J., M. B. Esch, R. P. Glahn, and M. L. Shuler. Characterization of a gastrointestinal tract microscale cell culture analog used to predict drug toxicity. Biotechnol. Bioeng. 104:193-205, 2009.

${ }^{14}$ Mahler, G. J., M. L. Shuler, and R. P. Glahn. Characterization of Caco-2 and HT29-MTX co-cultures in an in vitro digestion/cell culture model used to predict iron bioavailability. J. Nutr. Biochem. 20:494-502, 2009.

${ }^{15}$ Natarajan, A., M. Stancescu, V. Dhir, C. Armstrong, F. Sommerhage, J. J. Hickman, and P. Molnar. Patterned cardiomyocytes on microelectrode arrays as a functional, high information content drug screening platform. Biomaterials 32(18):4267-4274, 2011.

${ }^{16}$ Shuler, M. L., S. Leung, and C. C. Dick. A mathematical model for the growth of a single bacterial cell. Ann. N.Y Acad. Sci. 326:35-56, 1979.

${ }^{17}$ Sin, A., K. C. Chin, M. F. Jamil, Y. Kostov, G. Rao, and M. L. Shuler. The design and fabrication of three-chamber microscale cell culture analog devices with integrated dissolved oxygen sensors. Biotechnol. Prog. 20:338-345, 2004.

${ }^{18}$ Sung, J. H., J. R. Choi, D. H. Kim, and M. L. Shuler. Fluorescence optical detection in situ for real-time monitoring of enzymatic activity of liver cells in multiple microfluidic devices. Biotechnol. Bioeng. 104:516-525, 2009.

${ }^{19}$ Sung, J. H., C. Kam, and M. L. Shuler. A microfluidic device for pharmacokinetic-pharmacodynamic (PK-PD) model on a chip. Lab Chip 10:446-455, 2010.

${ }^{20}$ Sung, J. H., and M. L. Shuler. A micro cell culture analog (microCCA) with 3-D hydrogel culture of multiple cell lines to assess metabolism-dependent cytotoxicity of anti-cancer drugs. Lab Chip 9:1385-1394, 2009.

${ }^{21}$ Sung, J. H., and M. L. Shuler. Prevention of air bubble formation in a microfluidic perfusion cell culture system using a microscale bubble trap. Biomed. Microdevices 11:731-738, 2009.

${ }^{22}$ Sung, J. H., and M. L. Shuler. In vitro microscale systems for systematic drug toxicity study. Bioprocess Biosyst. Eng. $33: 5-19,2010$

${ }^{23}$ Sung, J. H., J. Yu, D. Luo, M. L. Shuler, and J. C. March. Microscale 3-D hydrogel scaffold for biomimetic gastrointestinal (GI) tract model. Lab Chip 11:389-392, 2011.

${ }^{24}$ Sweeney, L. M., M. L. Shuler, J. G. Babish, and A. Ghanem. A cell culture analog of rodent physiology: application to naphthalene toxicology. Toxicol. In Vitro 9(3):307-316, 1995. 
${ }^{25}$ Tatosian, D. A., and M. L. Shuler. A novel system for evaluation of drug mixtures for potential efficacy in treating multidrug resistant cancers. Biotechnol. Bioeng. 103:187-198, 2009.

${ }^{26}$ Tsuchiya, H. M., A. G. Frederickson, and R. Aris. Dynamics of microbial cell populations. Adv. Chem. Eng. 6:125-206, 1966.
${ }^{27}$ Viravaidya, K., and M. L. Shuler. Incorporation of 3T3-L1 cells to mimic bioaccumulation in a microscale cell culture analog device for toxicity studies. Biotechnol. Prog. 20:590597, 2004

${ }^{28}$ Viravaidya, K., A. Sin, and M. L. Shuler. Development of a microscale cell culture analog to probe naphthalene toxicity. Biotechnol. Prog. 20:316-323, 2004. 